\title{
Recurrence plots for dynamic analysis of Type-I ELMs at JET with a Carbon wall
}

\author{
Barbara Cannas, Alessandra Fanni, Andrea Murari, Fabio Pisano and JET Contributors
}

\begin{abstract}
In this paper, the dynamic characteristics of Type-I ELM time series from the JET tokamak, the world's largest magnetic confinement plasma physics experiment, have been investigated through recurrence plots. The analysis has been focused on Recurrence Plots of pedestal temperature, line averaged electron density and outer divertor $D_{\alpha}$ time series during experiments with a carbon wall. The analysis of Recurrence Plots shows patterns similar to those characteristic of signals exhibiting type-2 intermittency, in particular, a characteristic kite-like shape; this gives useful hints to model the temperature signal as well as the $D_{\alpha}$ radiation time series, with simple nonlinear maps capturing the nearly periodic behaviour of Type-I ELMs.
\end{abstract}

Index Terms-Edge Localized Modes, Recurrence Plots, ELM modelling

\section{INTRODUCTION}

$\mathrm{I}^{\mathrm{N}}$ 1982 it was first observed at the Axially Symmetric Divertor Experiment (ASDEX) tokamak, that externally heated tokamak plasmas can rapidly reach an operating regime of improved confinement. The operating regime transition is normally accompanied by appearance of recurrent magnetohydrodynamic instabilities, known as Edge Localized Modes (ELMs). ELMs manifest themselves as short repetitive bursts of energy and particles at the plasma edge. The loss of energy and particles, which flow along the magnetic field lines towards the divertor plates, deteriorates the confinement, which may cause damage to the first wall. Additionally, the short but intense particle and power loads on the divertor cause erosion of the plates, which might become a serious concern in future machines, such as the International Thermonuclear Experimental Reactor, ITER. Despite these drawbacks, ELMs are beneficial in expelling exhaust impurities and helium ash that otherwise would accumulate in the plasma and eventually terminate the fusion burn. They also provide a means for density control. Hence the understanding and control of the level and nature of the ELM activity is crucial for the achievement of fusion power.

Although a number of ELM types have been classified, the

Manuscript submitted June 25, 2018. This work has been carried out within the framework of the EUROfusion Consortium and has received funding from the Euratom research and training programme 2014-2018 under grant agreement No 633053. The views and opinions expressed herein do not necessarily reflect those of the European Commission. The authors would like also to thank the following colleagues for their technical support in the data validation process: Paulo Abreu, Alexandru Boboc, Lucy Kogan.

B. Cannas, A. Fanni and F. Pisano are with the Department of Electrical and Electronic Engineering, University of Cagliari, via Marengo 2, 09123 Cagliari, physics of ELMs is still to a large extent unresolved. The information about the ELM dynamics is fundamental to develop a proper dynamical model, which could provide guidance in mitigating ELMs in view of the steady state operation of ITER. Several attempts are reported in literature to identify the nature, stochastic or deterministic, of ELMs and the results are sometimes conflicting. In [1] the results of a nonlinear dynamic analysis of Type-I ELM time series excluded the presence of chaos leading to the hypothesis of pseudoperiodic behaviour.

In this paper, the dynamic characteristics of Type-I ELM time-series from the JET tokamak, the world's largest magnetic confinement plasma physics experiment, have been investigated through Recurrence Plots (RPs). This tool, together with the Recurrence Quantification Analysis (RQA), is a modern method of nonlinear data analysis. The tool of RPs, introduced in 1987 by Eckmann et al. [2], allows the visualization of a square matrix in which the matrix elements correspond to those times at which a state of a dynamical system recurs, revealing if the phase space trajectory of the dynamical system visits roughly the same area. The advantage of RPs is that they can also be applied to rather short and even non-stationary data.

A huge amount of data for each JET experiment is available, up to several tens of GB. Time series of relevant plasma parameters are stored in the JET data warehouse. For ELM studies, the analysis has been focused on pedestal temperature, electron density at the edge of the plasma, and outer divertor deuterium visible light emission $\left(\mathrm{D}_{\alpha}\right)$ time series.

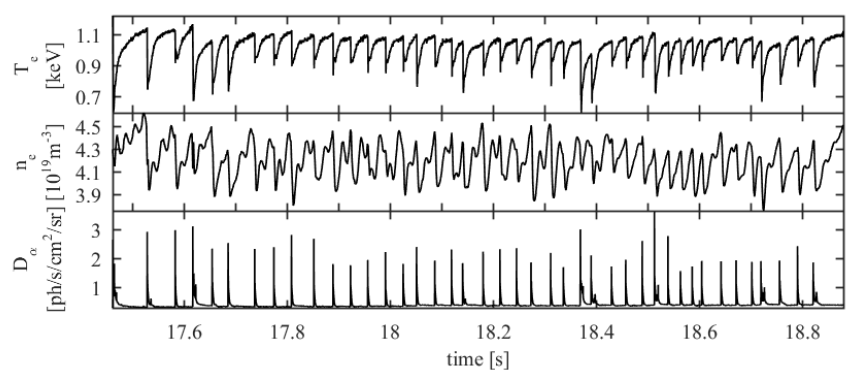

Fig. 1. Pedestal temperature (top), line averaged electron density at the edge (middle), and $\mathrm{D}_{\alpha}$ (bottom) time series for shot 74444 .

Italy_ (e-mail: cannas@diee.unica.it, fanni@diee.unica.it, fabio.pisano@diee.unica.it).

A. Murari is with Consorzio RFX, EURATOM-ENEA Association, Corso Stati Uniti 4, 35127 Padova, Italy (e-mail: Andrea.Murari@euro-fusion.org).

For JET Contributors see the author list of "Overview of the JET results in support to ITER" by X. Litaudon et al. to be published in Nuclear Fusion Special issue: overview and summary reports from the 26th Fusion Energy Conference (Kyoto, Japan, 17-22 October 2016) 
Fig. 1 shows the pedestal temperature, the line averaged electron density at the edge, and the $D_{\alpha}$ time series for shot 74444 .

All temperature, density and $\mathrm{D}_{\alpha}$ time series exhibit early regular behaviour (laminar flow) intermittently interrupted by outbreaks (bursts) at irregular intervals. From a physical point of view, the ELM cycle consists of two phases: an ELM crash due to the MHD instability inducing energy and particle losses from the pedestal, and a quiescent phase recovering the pedestal pressure between ELMs (inter-ELM phase). The delay period between ELMs is apparently random; for this reason the nature of the irregularities is still under debate and the literature reports several attempts to establish if the ELM nature is deterministic or stochastic [3-9]. As it can be noticed, each ELM cycle is characterized in all the time series by asymmetry, i.e. temperature and density decrease ( $\mathrm{D}_{\alpha}$ increases) more rapidly than they increase (it decreases). This type of behaviour is typical of intermittent systems [10]. Such signals are copious in natural and physiological systems: e.g., annual sunspot numbers [11-12], laser output [13-17], human electrocardiogram [18-19], etc..

The paper is organized as follows: section II describes the RPs; section III presents the database; section IV reports the results of the analysis; in section $\mathrm{V}$ the conclusions are drawn.

\section{RECURRENCE PLOTS}

Let us consider a dynamical system

$$
\frac{d \boldsymbol{x}(t)}{d t}=f(\boldsymbol{x})
$$

and a scalar observable $y(t)$. The first step to build a recurrence plot is to reconstruct the dynamics by embedding the onedimensional time series $y(t)$ in a $d$-dimensional space using the method of delay coordinates [20]. The general topological result of Mañé and Takens [21-22] states that the complete dynamics of a system can be reconstructed from a set $\boldsymbol{y}_{n}=\left[y_{n-}\right.$ $\left.(m-1) \tau, y_{n-(m-2) \tau}, \ldots, y_{n-\tau}, y_{n}\right]$ of time delayed versions of a suitable scalar measurement $y_{n}$ derived from the system at multiples of a fixed sampling time, where the embedding dimension $m$ and the time delay $\tau$ are two critical parameters. Takens and Mañé proved that, if $m \geq 2 D+1$, where $D$ is the box counting dimension of the attractor, there exists a one to one correspondence between the state space reconstructed and the original one. This is a sufficient but not necessary condition. In practice, an attractor may also be reconstructed successfully with an embedding dimension satisfying $m \geq D$. The embedding dimension $m$ can be estimated through the method of False Nearest Neighbors (FNNs) [23]. A simple criterion to compute the time delay $\tau$ is to set it equal to the first minimum of the average mutual information $I_{\tau}$ [24], when present.

After reconstructing the embedding space, the RP can be mathematically expressed by a two-dimensional squared matrix, the so-called recurrence matrix

$$
\mathfrak{R}_{i, j}=\Theta\left(\varepsilon-\left\|\boldsymbol{y}_{i}-\boldsymbol{y}_{j}\right\|\right), \quad \boldsymbol{y}_{i}, \boldsymbol{y}_{j} \in \mathbb{R}^{m}, \quad i, j=0, \ldots, N-1
$$

where $N$ is the number of considered samples $\boldsymbol{y}_{i}, \varepsilon$ is a threshold distance, $\|\cdot\|$ is a norm and $\Theta(\cdot)$ is the Heaviside function. Each element of the matrix represents the recurrence at a different time $j$ of a state at time $i$, equal to one if the distance between the two states is lower than $\varepsilon$, and equal to zero otherwise. The $\mathrm{RP}$ is obtained by plotting the matrix in a square map where both axes are time axes and assigning black dots to ones and white dots to zeroes.

Since $\mathfrak{R}_{i, i}=1, \forall i$, the RP has always a black main diagonal line, named the line of identity (LOI). Furthermore, the RP is symmetric by definition with respect to the main diagonal $\left(\mathfrak{R}_{i, j}=\mathfrak{R}_{j, i}\right)$.

Iwanski and Bradley [25] proposed the unthresholded recurrence plot (UTRP), a variation of the RP which plots directly the distance matrix

$\Delta_{i, j}=\left\|\boldsymbol{y}_{i}-\boldsymbol{y}_{j}\right\|, \quad \boldsymbol{y}_{i}, \boldsymbol{y}_{j} \in \mathbb{R}^{m}, \quad i, j=0, \ldots, N-1$

By using an appropriate color bar for the values of the threshold distance $\varepsilon$ it is possible to highlight the different recurrence structures at different thresholds.

Iwanski and Bradley [30] found that the appearance and statistics of recurrence plots for certain low-dimensional systems are not significantly altered by a small change in the embedding dimension, suggesting that these statistics may be important new invariant characteristics of a system.

\section{THE ELM DATABASE}

In [26] a statistical analysis of 60 JET $D_{\alpha}$ signals characteristic of JET Type-I ELMs was performed. The reference experimental campaigns, all in Deuterium, were C21 - C27b (9th June 2008 - 23rd October 2010). The wall was in graphite. The analysis was restricted to time intervals with fixed engineering conditions: only the experiments and time intervals characterized by variations of $\pm 4 \%$ for toroidal magnetic field $B_{\tau}, \pm 2 \%$ for plasma current $I_{p}, \pm 10 \%$ for NBI input power $P_{N B I}$ and lower triangularity $\delta_{\text {low }}$, were taken into account. All tolerances were related to the signal resolutions. The 60 shots correspond to 24 experimental conditions with different values of $I_{p}, B_{\tau}, P_{N B I}$ and $\delta_{\text {low }}$ for a total of 3448 TypeI ELM time intervals.

After the localization of the inter-ELM time intervals, the memorylessness test suggested the presence of memory in the ELM time intervals for the considered Type-I ELMs, in agreement with previous studies [27-28]. From a statistical point of view, the probability distribution of inter-ELM periods did not show the same properties with varying the experimental conditions. Pulses relative to similar inputs were grouped into 24 groups, including 10 singles, 4 pairs and 10 cliques. KruskalWallis test [29] was applied to inter ELM intervals of pulses of the same group to verify whether they belonged to the same population. Four groups $(6,10,16,19)$ were identified with $5 \%$ confidence level (see Table I). The following analysis is restricted to pulses in these four groups. Nevertheless, the obtained results can be extended to all Type-I ELMs in the same plasma conditions ( $\mathrm{CW}$ wall, deuterium plasma) since they are obtained on the base of qualitative considerations on the signals shape.

The same database has been used in [1] to investigate the pseudo-periodicity of ELM time series.

In the next section, the previously described database is analysed with RPs; a subscript, which indicates the membership clique, is added to the name of each pulse for ease of notation.

TABLE I 
GROUPS WITH DIVISION BY ENGINEERING CONDITIONS (PLASMA CURRENT, TOROIDAL MAGNETIC FIELD, NBI INPUT POWER AND LOWER TRIANGULARITY) AND BELONGING TO THE SAME EXPERIMENT.

\begin{tabular}{|c|c|c|c|}
\hline & Pulses & $\begin{array}{l}\text { \# of } \\
\text { intervals }\end{array}$ & Engineering conditions \\
\hline 6 & 74375,74376 & 54 & $\begin{array}{l}2.5 \mathrm{MA}, 2.5 \mathrm{~T}, \\
15.1-15.5 \mathrm{MW}, 0.33-0.35\end{array}$ \\
\hline 10 & 74443,74444 & 90 & $\begin{array}{l}\text { 2.5MA, 2.7T, } \\
14-15.8 \mathrm{MW}, 0.32\end{array}$ \\
\hline 16 & $\begin{array}{l}76428,76430,76431, \\
76437,76438\end{array}$ & 197 & $\begin{array}{l}\text { 2MA, } 2 \mathrm{~T} \\
7.5 \mathrm{MW}, 0.35\end{array}$ \\
\hline 19 & $\begin{array}{l}76470,76471,76472, \\
76473,76474,76475, \\
76476,76477,76478, \\
76479\end{array}$ & 363 & $\begin{array}{l}2 \mathrm{MA}, 2 \mathrm{~T}, \\
14.5-16.8 \mathrm{MW}, 0.35-0.37\end{array}$ \\
\hline
\end{tabular}

\section{RESULTS}

\section{A. Recurrence plots}

The embedding parameters have been evaluated for temperature, density, and $\mathrm{D}_{\alpha}$ time series before creating the RPs. If we consider that the rise/crash time of a burst is about 2-3 sampling times, it can be assumed that a small $\tau$ is necessary. Fig. 2 shows that, with $\tau=1$, the attractor is well unfolded. Thus, the chosen value is $\tau=1$.

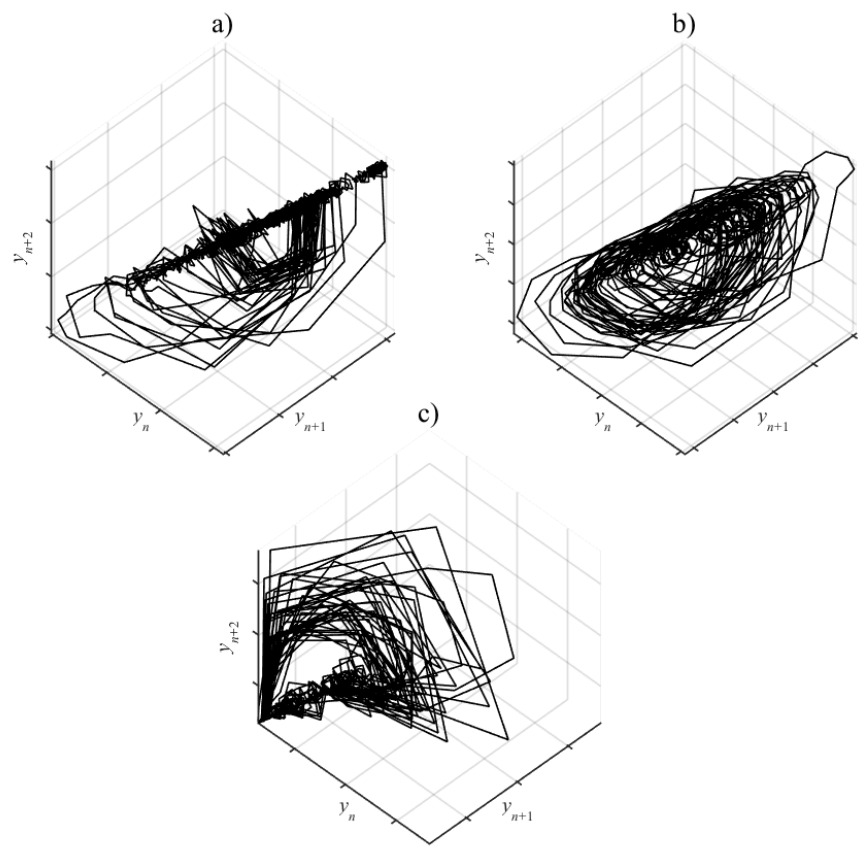

Fig. 2. 3D Projection for a) pedestal temperature, b) density at the edge and c) outer divertor $\mathrm{D}_{\alpha}$, with $\tau=1$

To determine the appropriate embedding dimension, the False Nearest Neighbours (FNNs) method has been used. The estimated embedding dimension could be evaluated as the shortest $m$ for which the percentage of FNNs is lower than $30 \%$. The obtained results on the list of shots showed $m=3$ for the temperature and density time series and values in the interval 4 -7 for the $\mathrm{D}_{\alpha}$ time series. It is worth noting that this estimation might be greater than the right embedding dimension due to the presence of noise in the signals. Fortunately, RPs are not particularly sensitive to the choice of embedding parameters and we have found only marginal, almost invisible, differences when choosing embedding dimension and time delay within reasonable ranges.

In Fig. 3a the UTRP related to temperature time series for shot $76471_{19}$ is shown, where black and white points represent respectively the smallest and the highest distances between points. White corridors are present when ELMs appear, i.e. the distance between points of the burst and those of the laminar phase is high. Squares or rectangular blocks among white corridors correspond to laminar phases. By introducing the threshold $\varepsilon$, the shape of the blocks varies (Fig. 3b). The RPs of the temperature time series have a checkerboard structure, which is typical of periodic and quasi-periodic systems [30]. In particular, for $\varepsilon$ chosen such that the recurrence point density is approximately $30 \%$, squares and rectangles with an elongated lower left corner are present. Moreover, the presence of close secondary peaks gives rise to larger and closer white corridors in the UTRP and white corridors in the central region of the RP.

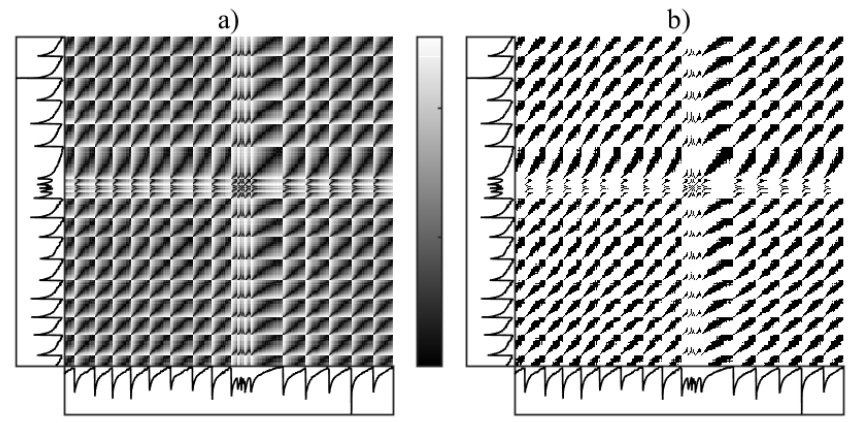

Fig. 3. a) UTRP and b) RP for temperature time series of shot $76471_{19}$.

The UTRP and RP in Fig. 4 for shot $74375_{6}$ highlight the presence of non-stationarity. Indeed, large bright regions on the higher left and lower right part give evidence of increasing distances among points of the first and second part of the series in the embedding space, harder to notice from the visualization of the time series alone. This is due to the fact that the second part of the time series is characterized by slightly higher values during the laminar phase.
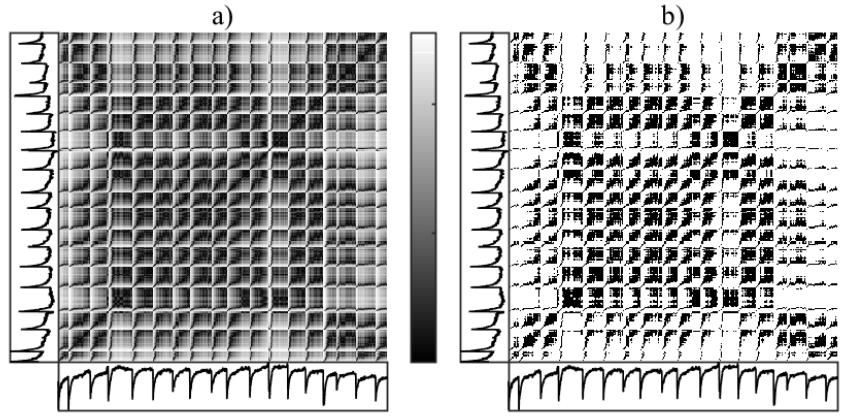

Fig. 4. a) UTRP and b) RP for temperature time series of shot $74375_{6}$.

Fig. 5-6 show the UTRP and RP of density and $\mathrm{D}_{\alpha}$ time series for shot $74375_{6}$. As it can be noticed easily, the same typical kite-like shape appears in the RP image, although the effect of non-stationarity is less visible, both for density and $D_{\alpha}$. Moreover, the RP for density shows a more complex structure inside the kite, mainly due to the high frequency oscillations in the time series. 


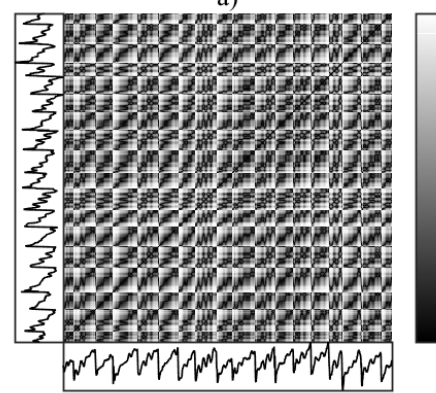

b)

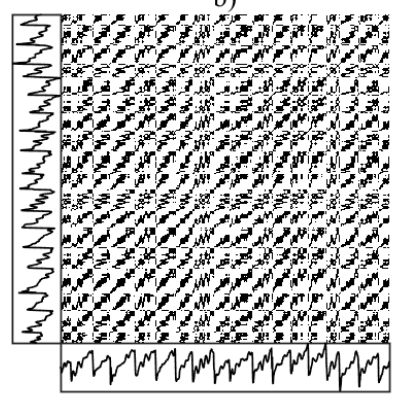

Fig. 5. a) UTRP and b) RP for density time series of shot $74375_{6}$.

a)

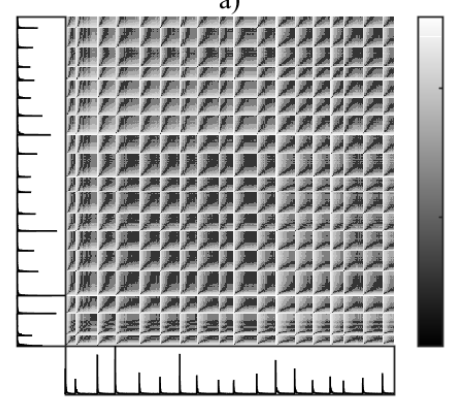

Fig. 6. a) UTRP and b) RP for $\mathrm{D}_{\alpha}$ time series of shot $74375_{6}$.

\section{B. From type-2 Intermittency to ELMs}

As stated above, the shape of squares and rectangles with an elongated lower left corner resembles a uniformly black kite. This is a characteristic common to all the cliques. The same shape is characteristic of RPs for type-2 intermittency systems [31], model of weakly chaotic behavior. It is important to clarify that there is no connection between the intermittency type (type-2) and Type-II ELMs [32], which are not subject of the present paper.

One of the first models of type-2 Intermittency, developed by Manneville in 1980 [33, 34], consists of a simple iteration for laminar and reinjection phases

$$
r_{n+1}= \begin{cases}f\left(r_{n}\right)=(1+\alpha) r_{n}+(1-\alpha) r_{n}^{2} & r_{n} \leq r_{t h} \\ f\left(r_{n}\right)-1 & r_{n}>r_{t h}\end{cases}
$$

where $r_{t h}$ is chosen such that $f\left(r_{t h}\right)=1$. System (9), when $\alpha>0$, shows ordinary chaotic dynamics. Fig. 7 shows the $r_{n}$ time series obtained for $\alpha=0.001$.

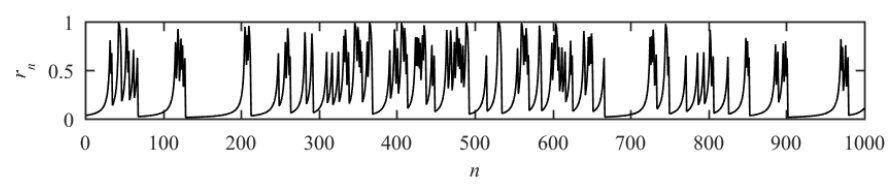

Fig. 7. Dynamics of type-2 intermittency ( $\alpha=0.001)$.

The UTRP and RP for the $r_{n}$ time series are shown in Fig. 8. The threshold $\varepsilon$ of the RP has been chosen such that the recurrence plot density is approximately 30\%. A uniformly black kite-like shape appears for the laminar phase in the RP in Fig. 8b.
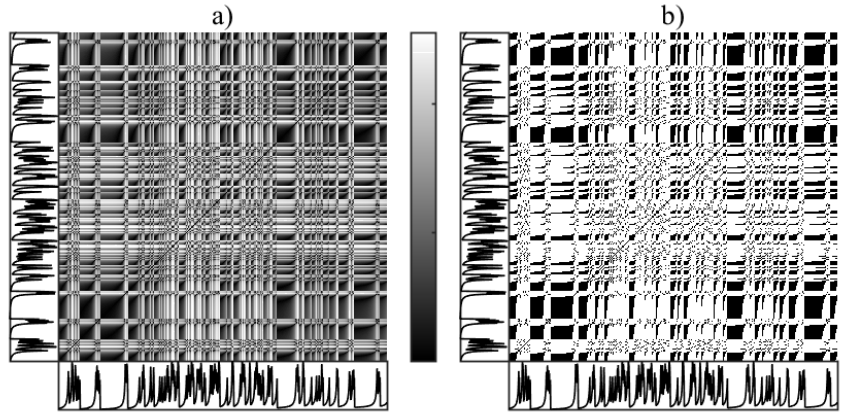

Fig. 8. a) UTRP and b) RP for type-2 intermittency time series $(\alpha=0.001)$.

There is an evident similarity between RPs of type-2 intermittency and those of temperature and $D_{\alpha}$ time series, which exhibit the same characteristic kite-like shape, despite the different orientation. This main difference is due to the fact that in the temperature $\left(D_{\alpha}\right)$ series, the growth (decrease) is first fast and then slows whereas $r_{n}$ is characterized by an initially slow growth which becomes faster with time and a following fast decay. The RPs similarity suggests further investigation of intermittent systems with the scope of modeling Type-I ELMs in terms of relaxation and drive during aperiodic oscillations. In this case, the aperiodic behavior is interpreted as a pseudoperiodic behavior, i.e., a periodic behavior corrupted by a dynamical noise [1],

As an attempt to obtain the same orientation of the kites in the RP, the map (9) has been inverted in the laminar region and its convexity modified to obtain a map which qualitatively describes the behavior of temperature time series, i.e., a slow growth towards an equilibrium value $T_{e q}$, interrupted by a fast crash after crossing a threshold $T_{t h}$ (see Fig. 9). Once this critical value has been exceeded, the system relaxes to a status of lower energy by rapidly decreasing the pressure gradient. Then the cycle repeats and can continue indefinitely if the conditions remain stationary. This kind of instability is guided by pressure, and the ELM frequency increases with the heating power. This translates into an inverse proportionality between the heating power and the mean laminar phase length.

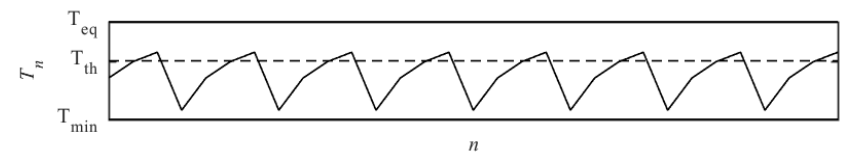

Fig. 9. Time series generated by inverting the map (9) in the laminar region.

The first equation describes the relaxation and dissipation of the perturbations and the second the drive process in the plasma. The model can be described by

$$
\begin{aligned}
& T_{n+1}= \begin{cases}h_{T}\left(T_{n}\right) & T_{\min } \leq T_{n} \leq T_{t h} \\
g_{T}\left(T_{n}\right) & T_{t h}<T_{n} \leq T_{\max }<T_{e q}\end{cases} \\
& h_{T}(T)=T_{e q}-f^{-1}\left(T_{e q}-T\right) \\
& g_{T}(T)=T_{\text {min }}+\gamma_{T} \frac{h_{T}(T)-h_{T}\left(T_{t h}\right)}{h_{T}\left(T_{\max }\right)-h_{T}\left(T_{t h}\right)}
\end{aligned}
$$

where $T_{\min }$ is the minimum value obtainable by $T_{n}$ during the drive phase, $T_{\max }=h_{T}\left(T_{t h}\right)$ is the maximum value obtainable by $T_{n}$ during the relaxation phase, and the parameter 
$\gamma_{T} \in\left(0, T_{\max }-T_{\min }\right]$ is an arbitrary value which determines the maximum temperature attainable during the drive process.

The map in (10) exhibits different dynamic behaviors, depending on the parameters $\gamma_{T}$ and $T_{t h}$, both chaotic and periodic (see fig. 10a when $T_{\min }=0$ and $T_{e q}=1$ ). When the map is periodic, the period of the time series can vary between one and many ELM cycles. In fig. 10b the different dynamical behaviors are shown, where $N$ is the number of ELM cycles inside one period of the time series.
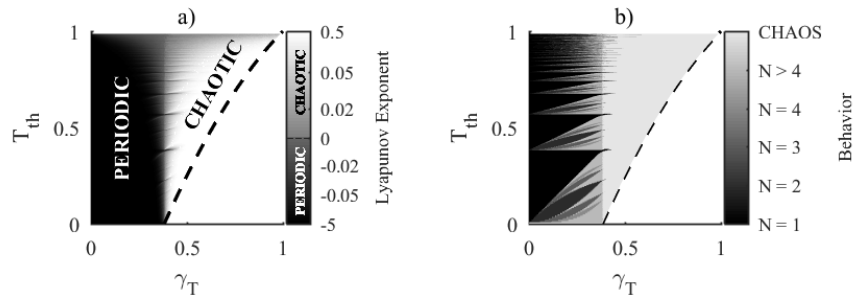

Fig. 10. a) Lyapunov Exponent and b) dynamical behavior for temperature model in (10) with varying parameters $\gamma_{T}$ and $T_{t h}(\alpha=0.001)$.

In particular, when $T_{e q}=1, T_{t h}=0.9793$ and $T_{\min }=0$, the map is periodic for $\gamma_{T}<0.3821$ and chaotic for $\gamma_{T}>0.3821$. When the map is periodic, $N=1$ for $\gamma_{T}<0.316$ and $N>1$ for $\gamma_{T}>0.316$. A property of the map, common to all values of $T_{t h}$, is that for low values of $\gamma_{T}$ the system is periodic with period equal to one ELM cycle; then, after increasing the value of $\gamma_{T}$, the map is subject to a continuous series of bifurcations that alter the size of the period, until the system becomes chaotic. Fig. 11 shows three time series, respectively obtained when $\gamma_{T}=0.2,0.35$ and 0.7 .

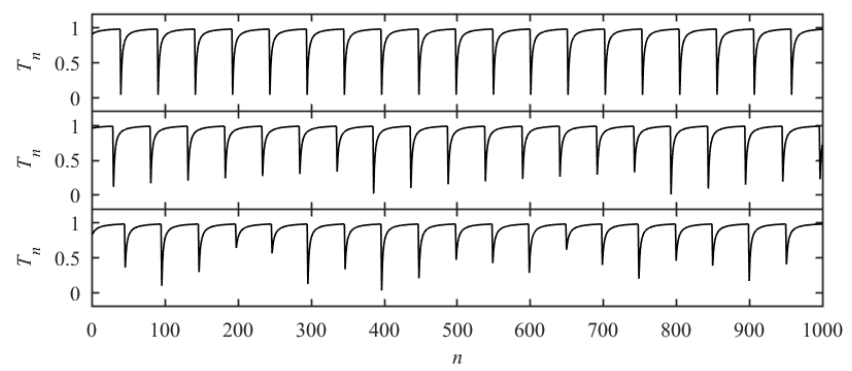

Fig. 11. Temperature-like time series for $T_{e q}=1, T_{t h}=0.9793, T_{\min }=0$. a) $\gamma_{T}=0.1$ (periodic, $N=1$ ); b) $\gamma_{T}=0.35$ (periodic, $N=8$ ); c) $\gamma_{T}=0.7$ (chaotic).

The system in (10) can exhibit also a pseudo-periodic behavior [1] when in the periodic region is corrupted by a dynamical noise. In this case, the resulting system is described by

$T_{n+1}=\eta_{n+1}+\left\{\begin{array}{ll}h_{T}\left(T_{n}\right) & T_{\min }-\eta_{\max } \leq T_{n} \leq T_{t h} \\ g_{T}\left(T_{n}\right) & T_{t h}<T_{n} \leq T_{\max }+\eta_{\max }\end{array}\right.$.

where the dynamic noise $\eta_{n}$ is uniform in the interval $\left[-\eta_{\max }, \eta_{\max }\right]$ and is responsible of the irregular behavior in frequency and amplitude of the signal.

A map, which graphically represents the behavior of the model in (11), is shown in Fig. 12. The gray stripes indicate the tolerances due to noise.

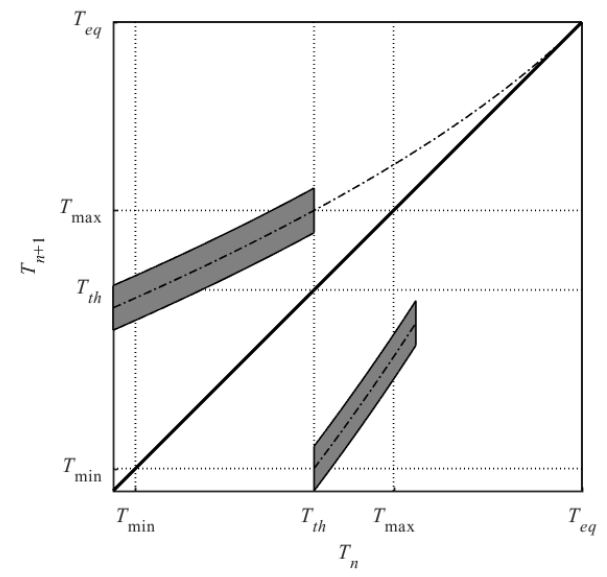

Fig. 12. Map for pseudo-periodic temperature model.

Fig. 13 shows the $T_{n}$ time series obtained for $\alpha=0.001$, $T_{\text {th }}=0.9793, T_{e q}=1, T_{\min }=0, \eta_{\max }=0.003, \gamma_{T}=0.1$.

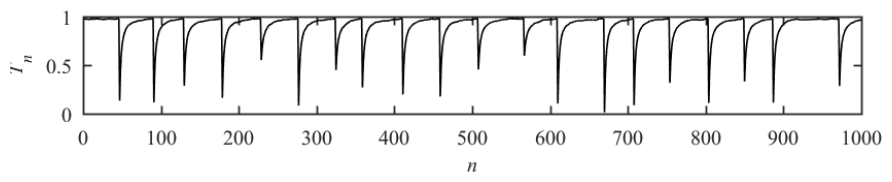

Fig. 13. Pseudo-periodic temperature-like time series.

The RP and UTRP for the obtained time series are shown in Fig. 14. The threshold $\varepsilon$ of the RP has been chosen such that the recurrence point density is approximately $30 \%$. A uniformly black kite-like shape appears for the laminar phase oriented as in temperature time series.

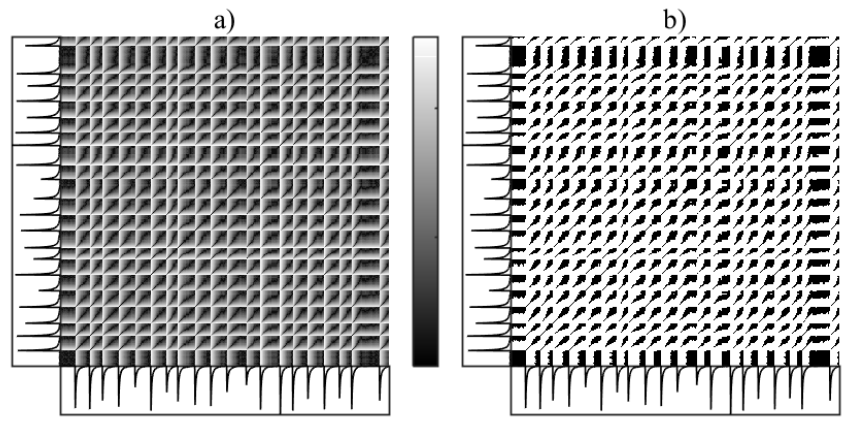

Fig. 14. a) UTRP and b) RP for temperature-like time series.

With respect to type-2 intermittency, the inversion and the convexity modification along the laminar phase changes the equilibrium point and its stability. In fact, in type-2 intermittency there is a single unstable equilibrium point in the origin, which destabilizes the trajectory, and a reinjection phase, which reinjects the trajectory in a zone close to the equilibrium point. In the new map there is a single stable equilibrium point in $T_{e q}$ towards which the trajectory tends and a crash phase in which the trajectory is sent far from the equilibrium point.

Moreover, with an analogue procedure, it is possible to reproduce a behavior similar to the one characteristic of the $\mathrm{D}_{\alpha}$ time series taking into account that the $\mathrm{D}_{\alpha}$ burst (decay) occurs when the temperature crashes (increases). Thus, a $\mathrm{D}_{\alpha}$ model can 
be obtained by setting $T=T_{e q}+D_{e q}-D$ in (11) obtaining a model of the form:

$$
\begin{aligned}
& D_{n+1}=\eta_{n+1}+ \begin{cases}g_{D}\left(D_{n}\right) & D_{\min }-\eta_{\max } \leq D_{n}<D_{t h} \\
h_{D}\left(D_{n}\right) & D_{t h} \leq D_{n} \leq D_{\max }+\eta_{\max }\end{cases} \\
& g_{D}(D)=D_{\max }-\gamma_{D} \frac{h_{D}\left(D_{t h}\right)-h_{D}(D)}{h_{D}\left(D_{t h}\right)-h_{D}\left(D_{\min }\right)} \\
& h_{D}(D)=D_{e q}+f^{-1}\left(D-D_{e q}\right)
\end{aligned}
$$

where $D_{\min }=h_{D}\left(D_{t h}\right)$ is the minimum value obtainable by $D_{n}$ during the relaxation phase when $\eta_{\max }=0, D_{\max }$ is the maximum value obtainable by $D_{n}$ during the drive phase when $\eta_{\max }=0$, $D_{e q}$ is the equilibrium point towards which the trajectory tends during the relaxation phase, and $\gamma_{D}$ is an arbitrary value which determines the minimum value reachable during the drive process.

A map which graphically represents the behavior of the model in (12) is shown in Fig. 15. The gray stripes indicate the tolerances due to noise.

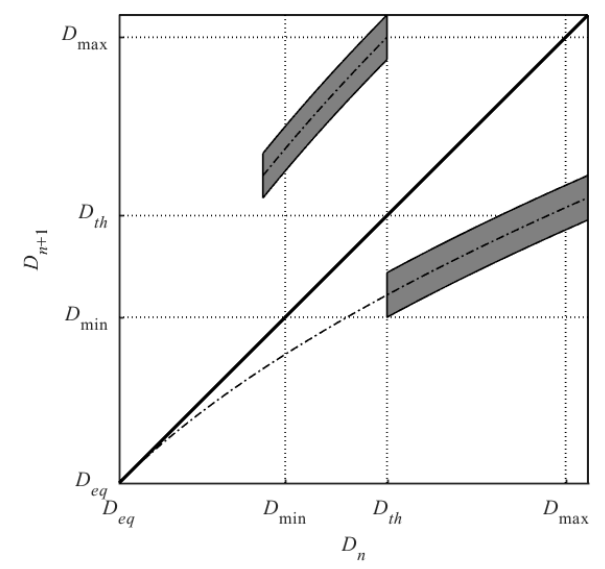

Fig. 15. Map for pseudo-periodic $\mathrm{D}_{\alpha}$ model.

Fig. 16 shows the $D_{n}$ time series obtained for $\alpha=0.001$, $D_{\text {th }}=0.0207, D_{\max }=1, D_{e q}=0, \eta_{\max }=0.003, \gamma_{D}=0.1$.

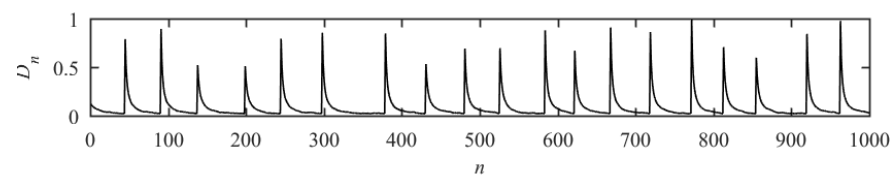

Fig. 16. Pseudo-periodic $\mathrm{D}_{\alpha}$-like time series.

The RP and UTRP for the obtained time series are shown in Fig. 17.
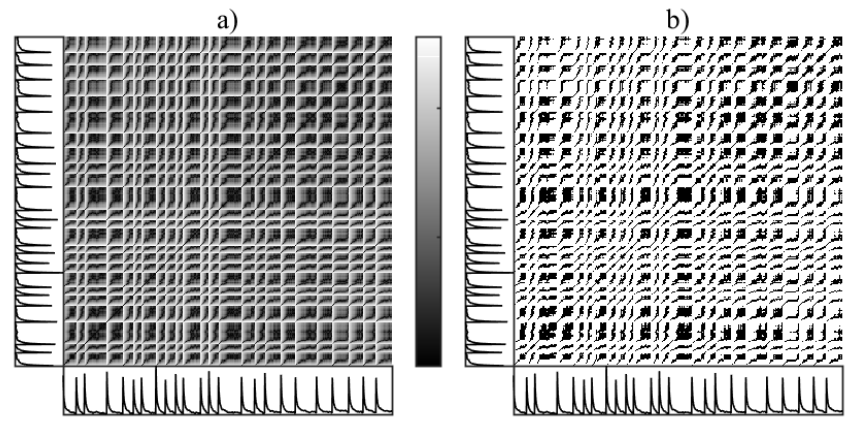

Fig. 17. a) UTRP and b) RP for $\mathrm{D}_{\alpha}$-like time series.
Also in this case a kite-like shape appears, similar to the one shown in $\mathrm{D}_{\alpha}$ and temperature time series.

\section{CONCLUSIONS}

Recurrence plots have been applied to investigate the characteristics of Type-I ELM time-series of JET tokamak with a carbon wall. Even if the discussion is developed at a heuristic level, avoiding all the delicate mathematical questions, we think it may shed some light on currently still puzzling problem of modeling ELMs.

Recurrence plots of temperature, density, and $\mathrm{D}_{\alpha}$ time series show patterns similar to those characteristic of signals exhibiting type-2 intermittency, in particular, a characteristic kite-like shape. Starting from this similarity, a qualitative model of temperature and $\mathrm{D}_{\alpha}$ time series has been derived starting from a type- 2 intermittency model. The models presented in the paper describe the qualitative behavior of temperature and $D_{\alpha}$ time series. Oscillations can be either periodic/pseudo-periodic with period varying between one and many ELM cycles, or chaotic: in case of temperature, the modelled time series is characterized by a slow growth towards an equilibrium value which is interrupted by a fast crash; for $D_{\alpha}$, the fast growth and the slower crash are reproduced.

This is a first attempt at modeling Type-I ELMs, which needs to be validated and connected with physical parameters and existing physical models. Firstly, a parameter identification will be made to fit the models to the individual signals in particular experimental conditions. The first objective of this identification phase is to find a connection between the threshold parameter in the model and the critical pressure gradient characteristic of the majority of physical models describing the ELM behavior.

\section{REFERENCES}

[1] B. Cannas, A. Fanni, A. Murari, F. Pisano and JET Contributors, "Nonlinear dynamic analysis of D $\alpha$ signals for type I edge localized modes characterization on JET with a carbon wall", Plasma Phys. Control. Fusion, vol. 60, no. 2, 025010, Dec 2017.

[2] J. P. Eckmann, S. O. Kamphorst, D. Ruelle, "Recurrence Plots of Dynamical Systems", Europhys. Lett., vol. 4, no. 9, pp. 973-977, Nov. 1987.

[3] P. E. Bak, R. Yoshino, N. Asakura, T. Nakano, "Identification of Unstable Periodic Orbit in Inter-Edge-Localized-Mode Intervals in JT-60U", Phys. Rev. Lett., vol. 83, no. 7, pp. 1339-1342, Aug. 1999.

[4] A. W. Degeling, Y. R. Martin, P. E. Bak, J. B. Lister, X. Llobet, "Dynamics of edge localized modes in the TCV tokamak", Plasma Phys. Control. Fus., vol. 43, no. 12, pp. 1671-1698, Oct. 2001.

[5] Y. R. Martin, A. W. Degeling, J. B. Lister, "Search for determinism in ELM time series in TCV", Plasma Phys. Control. Fus., vol. 44, no. 5A, A373-A382, Apr. 2002.

[6] G. Zvejnieks, V. N. Kuzovkov, O. Dumbrajs, A. W. Degeling, W. Suttrop, H. Urano, H. Zohm, "Autoregressive moving average model for analyzing edge localized mode time series on Axially Symmetric Divertor Experiment (ASDEX) Upgrade tokamak", Phys. Plasmas, vol. 11, no. 12, pp. 5658-5667, Nov. 2004.

[7] A. W. Degeling, J. B. Lister, Y. R. Martin, G. Zvejnieks, "Were the chaotic ELMs in TCV the result of an ARMA process?", Plasma Phys. Control. Fus., vol. 46, no. 10, pp. L15-L21, Sep. 2004.

[8] V. Hynonen, O. Dumbrajs, A.W. Degeling, T. Kurki-Suonio, H. Urano, "The search for chaotic edge localized modes in ASDEX Upgrade", Plasma Phys. Control. Fus., vol. 46, no. 9, pp. 1409-1422, Jul. 2004. 
[9] M. J. Ikonen, O. Dumbrajas, "Search for Deterministic Chaos in ELM Time Series of Asdex Upgrade Tokamak", IEEE Trans. Plasma Sci., vol. 33, no. 3, pp. 1115-1122, Jun. 2005.

[10] Y. Pomeau, P. Manneville, "Intermittent Transition to Turbulence in Dissipative Dynamical Systems", Commun. Math. Phys., vol. 74, no. 2, pp. 189-197, Jun. 1980.

[11] G. Gürbüz, C. Beck, "Sunspot activity curves and intermittent chaotic behaviour", Int. J. Bifurcat. Chaos, vol. 5, no. 4, pp. 1213-1219, Aug. 1995.

[12] E. A. Spiegel, "Chaos and Intermittency in the Solar Cycle", Space Sci. Rev., vol. 144, no. 1, pp. 25-51, Apr. 2009.

[13] D. Y. Tang, M. Y. Li, C. O. Weiss, "Laser dynamics of Type-I intermittency", Phys. Rev. A, vol. 46, no. 1, pp. 676-678, Jul. 1992.

[14] J. Pujol, M. Arjona, R. Corbalán, "Type-III intermittency in a four-level coherently pumped laser", Phys. Rev. A, vol. 48, no. 3, pp. 2251-2255, Sep. 1993.

[15] M. Arjona, J. Pujol, R. Corbalán, "Type-I intermittency in a four-level coherently pumped laser", Phys. Rev. A, vol. 50, no. 1, pp. 871 - 874, Jul. 1994.

[16] G. J. de Valcárcel, E. Roldán, V. Espinosa, R. Vilaseca, "Types I and II intermittencies in a cascade laser model", Phys. Lett. A, vol. 206, no. 5-6, pp. 359-364, Oct. 1995.

[17] C. Letellier, M. Bennoud, G. Martel, "Intermittency and period-doubling cascade on tori in a bimode laser model", Chaos Soliton. Fract., vol. 33, no. 3, pp. 782-794, Aug. 2007.

[18] J. J. Żebrowski, "Intermittency in human heart rate variability", Acta Phys. Pol. B, vol. 32, no. 5, pp. 1531-1540, May 2001.

[19] J. J. Żebrowski, R. Baranowski, "Type I intermittency in nonstationary systems-models and human heart rate variability", Phys. A, vol. 336, no. 1-2, pp. 74-83, May 2004.

[20] J. -P. Eckmann, D. Ruelle, "Ergodic theory of chaos and strange attractors", Rev. Mod. Phys., vol. 57, no. 3, pp. 617-656, Jul. 1985.

[21] R. Mañé, "On the dimension of the compact invariant sets of certain nonlinear maps", in: D. Rand, L.-S. Young, "Dynamical Systems and Turbulence”, Lect. Notes Math., vol. 898, pp. 230-242, Springer-Verlag, 1981.

[22] F. Takens, "Detecting strange attractors in turbulence", in: D. Rand, L.-S. Young, "Dynamical Systems and Turbulence", Lect. Notes Math., vol. 898, pp. 366-381, Springer-Verlag, 1981.

[23] M. B. Kennel, R. Brown, H. D. I. Abarbanel, "Determining embedding dimension for phase-space reconstruction using a geometrical construction", Phys. Rev. A, vol. 45, no. 6, pp. 3403-3411, Mar. 1992.

[24] A. Fraser, H. Swinney, "Independent coordinates for strange attractors from mutual information", Phys. Rev. A, vol. 33, no. 2, pp. 1134-1140, Feb. 1986.

[25] J. S. Iwanski, E. Bradley, "Recurrence plots of experimental data: To embed or not to embed?", Chaos, vol. 8, no. 4, pp. 861-871, Aug. 1998.

[26] A. Murari, F. Pisano, J. Vega, B. Cannas, A. Fanni, S. Gonzalez, M. Gelfusa, M. Grosso, JET EFDA Contributors, "Extensive statistical analysis of ELMs on JET with a carbon wall", Plasma Phys. Control. Fus., vol. 56, no. 11, p. 114007, Oct. 2014.

[27] A. J. Webster, R. O. Dendy, JET EFDA contributors, "Statistical Characterisation and Classification of Edge Localised Plasma Instabilities", Phys. Rev. Lett., vol. 110, no. 15, p. 155004, Apr. 2013.

[28] S. C. Chapman, R. O. Dendy, T. N. Todd, N. W. Watkins, F. A. Calderon, J. Morris, JET Contributors, "The global build-up to intrinsic edge localized mode bursts seen in divertor full flux loops in JET", Phys. Plasmas, vol. 22, no. 7, p. 072506, Jun. 2015.

[29] W. H. Kruskal, W. A. Wallis, "Use of ranks in one-criterion variance analysis", J. Am. Statist. Assoc., vol. 47, no. 260, pp. 583-621, Dec. 1952.

[30] J. P. Zbilut, C. L. Webber Jr., "Embeddings and delays as derived from quantification of recurrence plots", Phys. Lett. A, vol. 171, no. 3-4, pp. 199-203, Dec. 1992.

[31] K. Klimaszewska, J. J. Żebrowski, "Detection of the type of intermittency using characteristic patterns in recurrence plots", Phys. Rev. E, vol. 80 no. 2, p. 026214, Aug. 2009.

[32] J. W. Connor, "Edge Localized Modes - Physics and Theory", Plasma Phys. Control. Fus., vol. 40, no. 5, pp. 531-542, May 1998.

[33] P. Manneville, "Intermittency, self-similarity \& $1 \mathrm{f}$ spectrum in dissipative dynamical systems", J. Phys. France, vol. 41, no. 11, pp. 1235-1243, Nov. 1980.

[34] P. Manneville, Y. Pomeau, "Intermittent transition to turbulence in dissipative dynamical systems", Comm. Math. Phys., vol. 74, no. 2, pp. 189-197, Jun. 1980. 